\title{
Covariant Supplementation Scheme for Infinitely Reducible First Class Constraints
}

\author{
A.A. Deriglazov*and A.V. Galajinsky \\ Department of Theoretical Physics, Tomsk State University, 634050 \\ Tomsk, Russia
}

\begin{abstract}
For a rather broad class of dynamical systems subject to mixed fermionic first and second class constraints or infinitely reducible first class constraints (IR1C), a manifestly covariant scheme of supplementation of IR1C to irreducible ones is proposed. For a model with IR1C only, an application of the scheme leads to a system with covariantly splitted and irreducible first and second class constraints. Modified Lagrangian formulations for the Green-Schwarz superstring, CasalbuoniBrink-Schwarz superparticle and Siegel superparticle, which reproduce the supplementation scheme, are suggested.
\end{abstract}

PACS codes: $0460 \mathrm{D}, 1130 \mathrm{C}, 1125$.

Keywords: covariant quantization, superstring, superparticle.

The problem of constructing a covariant quantization scheme for dynamical systems with mixed first and second class constraints is extremely urgent since the Green-Schwarz (GS) superstring [1] and Casalbuoni-Brink-Schwarz (CBS) superparticle [2] belong to this class of theories.t A general recipe of Hamiltonian quantization without explicit splitting of the constraints has been developed in a series of works by Batalin and Tyutin [3]. However, as it was shown in the recent paper [4], an application of the scheme for concrete models may conflict with manifest Poincaré covariance.

An alternative possibility for the theories concerned consists in making use of covariant projectors to get splitted and reducible subsets of first and second class constraints. Projectors with desired properties have been constructed for the GS superstring [5], $D=9$ massive superparticle [6, 4], and $D=10, N=1 \mathrm{CBS}$ superparticle [7, 4]. This reduces the problem to quantization of linearly dependent second class constraints $(2 \mathrm{CC})$ (which can be treated in covariant fashion along the lines of Refs. 4, 7) and to quantization

\footnotetext{
${ }^{*}$ E-mail: deriglaz@phys.tsu.tomsk.su

${ }^{1}$ We mainly discuss the case of $D=10, N=1$ superspace for which there is no a Poincare covariant and irreducible splitting of the original fermionic constraints on first and second class in the initial phase space.
} 
of infinitely reducible first class constraints (IR1C). Unfortunately, the direct application of BFV-BV methods in the latter case leads to the formulations involving infinite extra ghost tower (see $[8,9]$ and references therein), what extremely complicates the analysis of BRST cogomologies and constructing effectively calculable quantum action.

In this letter, within the Hamiltonian and Lagrangian framework, we suggest a covariant scheme of supplementation of fermionic IR1C to a constraints system of finite stage of reducibility. In Hamiltonian approach, the initial phase space is enlarged by auxiliary variables, whose nondynamical character is provided by new reducible constraints. The proposed trick is based on a possibility to combine IR1C of extended formulation into covariant first class constraints system of finite stage of reducibility. After that, the standard quantization technique may be employed [10], in particular, with a finite number of ghost variables.

Two different cases will be considered: (i) models with mixed first and second class constraints; (ii) models with IR1C only (the latter situation takes place for modifications of the superstring and superparticle due to Siegel $[11,12]$ and their generalizations [7, 13]). In the first case, the resultant modified formulation contains irreducible first class constraints (1CC) and separated from them linearly dependent 2CC. In the second case, we shall get an extended system with irreducible $2 \mathrm{CC}$ and splitted from them $1 \mathrm{CC}$ no more than of first stage of reducibility. Although the presented scheme can be directly applied to constrained systems of special form only (see Eq. (1) below), a class of these theories is broad enough, in particular it includes all the above mentioned superstring and superparticle models.

In our opinion, the advantages of the proposed trick consist in the following: (i) Relatively small number of auxiliary variables are needed as compared to the combined harmonic-twistor approaches [14-16]. Note also that no twistor-like variables are introduced. (ii) There exists a covariant gauge for both the initial and auxiliary variables (compare with Refs. 14 and 17).

A consistent treatment of deformed constraints system implies the construction of modified Lagrangian formulation on enlarged configuration space which will reproduce the supplementation scheme. The existence of such a formulation will allow, in particular, to prove an equivalence of modified and initial models. The corresponding Lagrangian formulations for the GS superstring, CBS superparticle, and Siegel superparticle are presented and analyzed in the letter.

We work in 16-component formalism of the Lorentz group $S O(1,9)$, then $\theta^{\alpha}, \psi_{\alpha}$, $\alpha=1, \ldots, 16$, are Majorana-Weyl spinors of opposite chirality. Real, symmetric $16 \times 16$ $\Gamma$-matrices $\Gamma_{\alpha \beta}^{\mu}, \tilde{\Gamma}^{\mu \alpha \beta}$ obeying the algebra $\Gamma^{\mu} \tilde{\Gamma}^{\nu}+\Gamma^{\nu} \tilde{\Gamma}^{\mu}=-2 \eta^{\mu \nu}$ will be used. Momenta conjugate to configuration space variables $c^{i}$ are denoted as $p_{c i}$.

Let us consider a dynamical system with fermionic pairs $\left(\theta^{\alpha}, p_{\theta \alpha}\right)$ being presented among the phase space variables $z^{A}$. It is supposed that a complete constraints system of the model includes (among others) the following:

$$
L_{\alpha} \equiv p_{\theta \alpha}-i B_{\mu} \Gamma_{\alpha \beta}^{\mu} \theta^{\beta} \approx 0, \quad D^{\mu} D_{\mu} \approx 0
$$


and the Poisson bracket of the fermionic constraints is

$$
\left\{L_{\alpha}, L_{\beta}\right\}=2 i D_{\mu} \Gamma^{\mu}{ }_{\alpha \beta} .
$$

Here, the $B^{\mu}(z)$ and $D^{\mu}(z)$ are some functions of phase variables so that $D^{2} \approx 0$ is first class constraint. The full set of constraints of the theory may include ones different from Eq. (1), which are inessential for subsequent analysis. Note that Eqs. (1), (2) correspond to the CBS superparticle if we choose $B^{\mu}=D^{\mu}=p^{\mu}$, where $p^{\mu}$ are momenta conjugated to space-time coordinates $x^{\mu}$. To get the GS superstring case we choose $B^{\mu} \equiv p^{\mu}+\partial_{1} x^{\mu}-i \theta \Gamma^{\mu} \partial_{1} \theta, D^{\mu} \equiv p^{\mu}+\partial_{1} x^{\mu}-2 i \theta \Gamma^{\mu} \partial_{1} \theta$ (see below). From Eqs. (1), (2) it follows that there are eight $1 \mathrm{CC}$ and eight $2 \mathrm{CC}$ among the equations $L_{\alpha} \approx 0$. To separate them in a manifestly covariant fashion, let us extend the initial phase space by a pair of vector variables $\left(\Lambda^{\mu}, p_{\Lambda \mu}\right)$ subject to constraints

$$
\Lambda^{2} \approx 0, \quad p_{\Lambda}^{\mu} \approx 0 .
$$

Supposing that $\Lambda D \neq 0$ (analog of the standard light-cone singularity), one can extract two $2 \mathrm{CC}$ from Eq. (3): $\Lambda^{2} \approx 0, p_{\Lambda} D \approx 0$ and nine 1CC: $\tilde{p}_{\Lambda}^{\mu} \equiv p_{\Lambda}^{\mu}-\frac{p_{\Lambda} D}{\Lambda D} \Lambda^{\mu} \approx 0$ (there is identity $\left.D_{\mu} \tilde{p}_{\Lambda}^{\mu} \equiv 0\right)$. Thus, Eq. (3) provides a nondynamical character of the auxiliary variables. Since by construction $\left(\Lambda_{\mu}+D_{\mu}\right) \Gamma_{\alpha \beta}^{\mu}$ is a nondegenerate matrix, the constraints $L_{\alpha} \approx 0$ are equivalent to

$$
\begin{aligned}
& L^{(1) \alpha} \equiv D_{\mu} \tilde{\Gamma}^{\mu \alpha \beta} L_{\beta} \approx 0, \\
& L^{(2) \alpha} \equiv \Lambda_{\mu} \tilde{\Gamma}^{\mu \alpha \beta} L_{\beta} \approx 0,
\end{aligned}
$$

where among $1 \mathrm{CC} L^{(1)} \approx 0$ and $2 \mathrm{CC} L^{(2)} \approx 0$ there are in eight linearly independent. To supplement the IR1C in Eq. (4) to irreducible, let us further introduce a pair of spinor variables $\left(\chi^{\alpha}, p_{\chi \alpha}\right)$ subject to constraints[

$$
p_{\chi \alpha} \approx 0, \quad T_{\alpha} \equiv \Lambda_{\mu} \Gamma_{\alpha \beta}^{\mu} \chi^{\beta} \approx 0 .
$$

These equations contain 8 independent $1 \mathrm{CC}$ among $p_{\chi}^{(1)} \equiv \Lambda_{\mu} \tilde{\Gamma}^{\mu} p_{\chi} \approx 0$ and $8+8$ independent $2 \mathrm{CC}$ among $p_{\chi}^{(2)} \equiv D_{\mu} \tilde{\Gamma}^{\mu} p_{\chi} \approx 0, \Lambda_{\mu} \Gamma^{\mu} \chi \approx 0$. Note that the covariant gauge $D_{\mu} \Gamma^{\mu} \chi=0$ may be imposed after that the full system (constraints + gauge) is equivalent to $p_{\chi} \approx 0, \chi \approx 0$.

Within the framework of the extended formulation it is possible to combine part of the constraints into irreducible sets. Actually, taking into account that the matrix $\left(\Lambda_{\mu}+D_{\mu}\right) \Gamma^{\mu \alpha \beta}$ is nondegenerate one concludes that Eqs. (4) and (6) are equivalent to

$$
\begin{aligned}
& \Phi^{\alpha} \equiv L^{(1) \alpha}+p_{\chi}^{(1) \alpha}=D_{\mu} \tilde{\Gamma}^{\mu \alpha \beta} L_{\beta}+\Lambda_{\mu} \tilde{\Gamma}^{\mu \alpha \beta} p_{\chi \beta} \approx 0, \\
& G^{\alpha} \equiv L^{(2) \alpha}+p_{\chi}^{(2) \alpha}=\Lambda_{\mu} \tilde{\Gamma}^{\mu \alpha \beta} L_{\beta}+D_{\mu} \tilde{\Gamma}^{\mu \alpha \beta} p_{\chi \beta} \approx 0, \\
& T_{\alpha} \equiv \Lambda_{\mu} \Gamma^{\mu}{ }_{\alpha \beta} \chi^{\beta} \approx 0,
\end{aligned}
$$

${ }^{2}$ After that, instead of $\tilde{p}_{\Lambda}{ }^{\mu} \approx 0$, the following constraints:

$$
p_{\lambda}{ }^{\mu}-\frac{p_{\Lambda} D}{\Lambda D} \Lambda^{\mu}+\frac{1}{2 \Lambda D} p_{\chi} \tilde{\Gamma}^{\nu} D_{\nu} \chi \approx 0
$$

will be first class. 
where the $\Phi^{\alpha} \approx 0\left(G^{\alpha} \approx 0\right)$ are 16 irreducible $1 \mathrm{CC}(2 \mathrm{CC})$ and the $T_{\alpha} \approx 0$ include 8 linearly independent 2CC. As a result, for the modified formulation (3), (7)-(9) fermionic first and second class constraints are splitted in manifestly covariant fashion, so that $1 \mathrm{CC}$ are irreducible. Note that the situation with $2 \mathrm{CC}$ does not became "worse" as compared to the initial formulation. Some comments are in order.

(i) For the case of CBS superparticle constraints without light-cone singularities: $\Lambda^{2} \approx$ $0, \Lambda D-1 \approx 0, p_{\Lambda}^{\mu} \approx 0$ instead of Eq. (3) seem to be more suitable.

(ii) By making use of the vectors $D^{\mu}, \Lambda^{\mu}$ subject to constraints from Eqs. (1) and (3), the true projectors

$$
\begin{aligned}
& \Pi_{\alpha}^{ \pm}{ }_{\alpha}=\frac{1}{2}\left(1 \pm \frac{1}{2 b} \Gamma^{\mu \nu} D_{\mu} \Lambda_{\nu}\right)_{\alpha}{ }^{\beta}, \quad b=\sqrt{D^{2} \Lambda^{2}-(D \Lambda)^{2}}, \\
& 1=\Pi^{+}+\Pi^{-}, \quad\left(\Pi^{ \pm}\right)^{2}=\Pi^{ \pm}, \quad \Pi^{+} \Pi^{-}=0,
\end{aligned}
$$

can be constructed and applied in the previous scheme instead of the matrices $D_{\mu} \Gamma^{\mu}$, $\Lambda_{\mu} \Gamma^{\mu}$.

(iii) For some concrete models the described trick may be realized without introducing the variables $\left(\Lambda^{\mu}, p_{\Lambda \mu}\right)$. For the case of $D=9$ massive superparticle with WessZumino term there exists constant Lorentz-invariant matrix $z^{\alpha \beta}[4,6]$. It can be used for constructing the covariant projectors and splitting the constraints. For the case of GS superstring we may choose $\Lambda^{\mu} \equiv p^{\mu}-\partial_{1} x^{\mu}$, since $\left(p^{\mu}-\partial_{1} x^{\mu}\right)^{2} \approx 0$ is one of the super Virasoro constraints.

Proceeding to the case of models with IR1C only, let us suppose

$$
\begin{gathered}
L^{(1) \alpha} \equiv D_{\mu} \tilde{\Gamma}^{\mu \alpha \beta}\left(p_{\theta}-i B_{\mu} \Gamma^{\mu} \theta\right)_{\beta} \approx 0, \quad D^{\mu} D_{\mu} \approx 0 \\
\left\{L^{(1) \alpha}, L^{(1) \beta}\right\} \approx 0,
\end{gathered}
$$

instead of Eqs. (1), (2) (for instance, Siegel superparticle corresponds to the choice $D^{\mu}=$ $\left.B^{\mu}=p^{\mu}\right)$. Repeating the procedure described above, we get the following constraints system in extended by the variables $\left(\Lambda^{\mu}, p_{\Lambda \mu}\right)$ and $\left(\chi^{\alpha}, p_{\chi \alpha}\right)$ phase space:

$$
\begin{aligned}
& D^{2} \approx 0, \quad \Lambda^{2} \approx 0, \quad p_{\Lambda}^{\mu} \approx 0, \\
& \Phi^{\alpha} \equiv L^{(1) \alpha}+\Lambda_{\mu} \tilde{\Gamma}^{\mu \alpha \beta} p_{\chi \beta} \approx 0, \\
& p_{\chi}^{(2) \alpha} \equiv D_{\mu} \tilde{\Gamma}^{\mu \alpha \beta} p_{\chi \beta} \approx 0, \\
& T_{\alpha} \equiv \Lambda_{\mu} \Gamma_{\alpha \beta}^{\mu} \chi^{\beta} \approx 0 .
\end{aligned}
$$

In contrast to the previous case, it is impossible to combine 2CC from Eqs. (14), (15) in a manifestly covariant way because they belong to different inequivalent representations of $S O(1,9)$ group of opposite chirality. To avoid the problem, one needs further extension of the phase space in order to construct a matrix for lowering (raising) spinor indices. Let us introduce vectors $\left(C^{\mu}, p_{C}^{\mu}\right)$ subject to constraints

$$
C^{2}-1 \approx 0, \quad C \Lambda \approx 0, \quad C D \approx 0, \quad p_{C}^{\mu} \approx 0 .
$$


(Note that for all the above mentioned concrete models the condition $\left\{D^{2}, C^{\mu} D_{\mu}\right\} \sim$ $C^{\mu} D_{\mu}$ hold, and consequently the constraint $D^{2} \approx 0$ is first class as before.) The full system of bosonic constraints (13) and (16) can be splitted into first and second class sets

$$
\begin{aligned}
& \Lambda^{2} \approx 0, \quad p_{\Lambda} D \approx 0 ; \quad C^{2}-1 \approx 0, \quad p_{C} C \approx 0 \\
& C \Lambda \approx 0, \quad p_{C} D \approx 0 ; \quad C D \approx 0, \quad p_{C} \Lambda \approx 0 \\
& \tilde{p}_{\Lambda}^{\mu} \equiv p_{\Lambda}^{\mu}-\frac{p_{\Lambda} D}{\Lambda D} \Lambda^{\mu}-\left(p_{\Lambda} C\right) C^{\mu}+\frac{1}{2 \Lambda D} p_{\chi} \tilde{\Gamma}^{\nu} D_{\nu} \Gamma^{\mu} \chi \approx 0 \\
& \tilde{p}_{C}^{\mu} \equiv p_{C}^{\mu}-\frac{p_{C} D}{\Lambda D} \Lambda^{\mu}-\left(p_{C} C\right) C^{\mu}-\frac{p_{C} \Lambda}{\Lambda D} D^{\mu} \approx 0 \\
& \frac{p_{C} D}{\Lambda D}-p_{\Lambda} C+\frac{1}{2 \Lambda D} p_{\chi} C_{\nu} \tilde{\Gamma}^{\nu} \Gamma^{\mu} D_{\mu} \chi \approx 0
\end{aligned}
$$

where $1 \mathrm{CC}$ in Eq. (18) are first stage of reducibility. The reducibility is described by equations $\Lambda \tilde{p}_{\Lambda}=C \tilde{p}_{\Lambda}=\Lambda \tilde{p}_{C}=D \tilde{p}_{C}=C \tilde{p}_{C}=0$ which hold modulo 2CC (17). Manifestly covariant quantization of the sector (as well as Eq. (3)) may be carried out along the lines of Ref. 10.

Having in our disposal the invertible matrix $C_{\mu} \Gamma_{\alpha \beta}^{\mu}$, we pass from Eq. (14) to equivalent constraints

$$
p_{\chi \alpha}^{(2)} \equiv\left(C_{\mu} \Gamma^{\mu} D_{\nu} \tilde{\Gamma}^{\nu} p_{\chi}\right)_{\alpha} \approx 0,
$$

which may be covariantly combined now with Eq. (15). Thus, the resultant formulation being equivalent to Eqs. (11), (12) takes the form

$$
\begin{aligned}
& D^{2}=0, \quad \Lambda^{2}=0, \quad p_{\Lambda}^{\mu}=0, \\
& C^{2}=1, \quad C \Lambda=C D=0, \quad p_{C}^{\mu}=0, \\
& \Phi^{\alpha} \equiv L^{(1) \alpha}+\Lambda_{\mu} \tilde{\Gamma}^{\mu \alpha \beta} p_{\chi \beta}=0, \\
& G_{\alpha} \equiv \Lambda_{\mu} \Gamma_{\alpha \beta}^{\mu} \chi^{\beta}+\left(C_{\mu} \Gamma^{\mu} D_{\nu} \tilde{\Gamma}^{\nu} p_{\chi}\right)_{\alpha}=0,
\end{aligned}
$$

where the Poisson bracket of the constraints $(22)$ is $\left\{G_{\alpha}, G_{\beta}\right\}=-2(\Lambda D) C_{\mu} \Gamma_{\alpha \beta}^{\mu}$. As a result, the task of quantization of a model with infinitely reducible fermionic first class constraints has been reduced to quantization of splitted (in manifestly covariant way) and irreducible first and second class constraints (Eq. (21) and Eq. (22), respectively).

As an example of application the scheme proposed, we shall consider the GS superstring, CBS superparticle and Siegel superparticle. For each case, a modified action and its local symmetries in suitably enlarged configuration space will be presented. By passing from the Lagrangian formalism to the Hamiltonian one, we prove an equivalence of the modified and initial formulations and then demonstrate an applicability of the supplementation scheme.

$D=10, N=1$ Green-Schwarz superstring. Consider a covariant action of the form

$$
\begin{aligned}
& S=S_{\mathrm{GS}}+S_{\mathrm{add}}=\int d^{2} \sigma\left[-\frac{1}{2 \sqrt{-g}} g^{a b} \Pi_{a}^{\mu} \Pi_{b \mu}-i \varepsilon^{a b} \partial_{a} x_{\mu}\left(\theta \Gamma^{\mu} \partial_{b} \theta\right)-\right. \\
& \left.\quad-\frac{1}{2} \Lambda_{\mu} \varepsilon^{a b} F^{\mu}{ }_{a b}-\phi \Lambda^{\mu} \Lambda_{\mu}\right] \\
& \Pi_{a}{ }^{\mu} \equiv \partial_{a} x^{\mu}-i \theta \Gamma^{\mu} \partial_{a} \theta \\
& F^{\mu}{ }_{a b} \equiv \partial_{a} A_{b}{ }^{\mu}-\partial_{b} A_{a}{ }^{\mu}-i \partial_{a} \theta \Gamma^{\mu} \chi_{b}+i \partial_{b} \theta \Gamma^{\mu} \chi_{a}+i \chi_{a} \Gamma^{\mu} \chi_{b},
\end{aligned}
$$


where $S_{\mathrm{GS}}$ is the standard GS action [1]. It was chosen $\varepsilon^{01}=-1$, and the following auxiliary variables were introduced: scalar $\phi ; D=10$ vector $\Lambda^{\mu} ; D=2$ and $D=10$ vector $A_{a}{ }^{\mu} ; D=2$ vector and $D=10$ Majorana-Weyl spinor $\chi_{a}{ }^{\alpha}$. As will be seen, the only essential variables for the supplementation scheme are $A_{1}{ }^{\mu}$ and $\chi_{1}{ }^{\alpha}$, all other prove to supply $D=2$ reparametrization invariance of the action (23). Global symmetries of the theory (23) are standard $D=10, N=1$ super Poincaré transformations.

Local bosonic symmetries are $D=2$ reparametrizations, Weyl symmetry, and the following transformations with parameters $\xi^{\mu}(\sigma), \omega_{a}(\sigma)$ :

$$
\begin{aligned}
& \delta A_{a}{ }^{\mu}=\partial_{a} \xi^{\mu}+\omega_{a} \Lambda^{\mu}, \\
& \delta \phi=\frac{1}{2} \varepsilon^{a b} \partial_{a} \omega_{b} .
\end{aligned}
$$

These symmetries are reducible because their combination with parameters of a special form: $\omega_{a}=\partial_{a} \omega, \xi^{\mu}=-\omega \Lambda^{\mu}$, is a trivial symmetry: $\delta_{\omega} A_{a}{ }^{\mu}=-\omega \partial_{a} \Lambda^{\mu}, \delta_{\omega} \phi=0$ (note that $\partial_{a} \Lambda^{\mu}=0$ is one of the equations of motion). Thus, Eq. (24) includes 11 essential parameters which correspond to primary $1 \mathrm{CC} p_{A_{0}}^{\mu} \approx 0, p_{\phi} \approx 0$ (see below). Besides, there are local symmetries with fermionic parameters $\kappa^{-}{ }_{\alpha}^{a} \equiv P^{-a b} \kappa_{\alpha b}(\sigma)$ (where $P^{ \pm a b} \equiv$ $\left.\left(g^{a b} / \sqrt{-g} \pm \varepsilon^{a b}\right)\right)$ and $S^{+}{ }_{\alpha}{ }^{a}(\sigma)$ :

$$
\begin{aligned}
& \delta \theta=\Pi_{\mu a} \tilde{\Gamma}^{\mu} \kappa^{-a}, \\
& \delta x^{\mu}=i \theta \Gamma^{\mu} \delta \theta, \\
& \delta\left(\frac{g^{a b}}{\sqrt{-g}}\right)=4 i P^{-a c}\left(\partial_{c} \theta \kappa^{-b}\right), \\
& \delta \chi_{a}=\partial_{a}(\delta \theta)+\Lambda_{\mu} \tilde{\Gamma}^{\mu} \kappa^{-}{ }_{a}, \\
& \delta A^{\mu}{ }_{a}=i \theta \Gamma^{\mu} \partial_{a}(\delta \theta), \\
& \delta \phi=-i \epsilon^{a b}\left(\partial_{a} \theta-\chi_{a}\right) \kappa^{-}{ }_{b} ; \\
& \delta \chi_{a}{ }^{\alpha}=\Lambda_{\mu} \tilde{\Gamma}^{\mu \alpha \beta} S^{+}{ }_{\beta a}, \\
& \delta \phi=-i \varepsilon^{a b}\left(\partial_{a} \theta^{\alpha}-\chi_{a}{ }^{\alpha}\right) S^{+}{ }_{\alpha b} .
\end{aligned}
$$

Equation (25) is generalization of Siegel $\kappa$-symmetry [20] to the present case. In our formulation it is irreducible (with 16 essential parameters), and looks like a gauge symmetry (with the gauge field to be $\chi_{a}$, as is seen from its transformation law). The transformations (26) are reducible and incorporate only 8 essential parameters among the $S^{+}{ }_{\alpha}{ }^{a}$, because rank $\Lambda_{\mu} \Gamma^{\mu}=8$ on-shell, as a consequence of the equation of motion $\Lambda^{2}=0$. Note that it is not necessary to take care of the analog of the transformations (26) with the parameters $S^{-}{ }_{\alpha}^{a}$ since they have already been included into Eq. (25) [18]. Thus, the presented transformations with $16+8$ parameters exhaust all the essential fermionic symmetries of the model, because namely this number of primary fermionic $1 \mathrm{CC}$ will occur in the Hamiltonian formalism.

By direct application of the Dirac-Bergmann algorithm [19] one gets the Hamiltonian

$$
\begin{aligned}
H & =-\frac{1}{g^{00}}\left(\frac{\sqrt{-g}}{2}\left(\hat{p}^{2}+\Pi_{1}^{2}\right)-g^{01} \hat{p}_{\mu} \Pi_{1}^{\mu}\right)-\partial_{1} \Lambda_{\mu} A_{0}^{\mu}- \\
& -i \Lambda_{\mu}\left(\partial_{1} \theta-\chi_{1}\right) \Gamma^{\mu} \chi_{0}+\phi \Lambda^{2}+\lambda^{a b}\left(p_{g}\right)_{a b}+\lambda_{0} p_{\phi}+\lambda_{1 \mu} p_{\Lambda}^{\mu}+
\end{aligned}
$$




$$
+\lambda_{2 \mu} p_{A_{0}}^{\mu}+\lambda_{3 \mu}\left(p_{A_{1}}^{\mu}-\Lambda^{\mu}\right)+p_{\chi 0} \sigma_{0}+p_{\chi 1} \sigma_{1}+L \sigma_{3},
$$

where $\lambda, \sigma$ are Lagrange multipliers for primary constraints, and it was denoted $\hat{p}^{\mu} \equiv$ $p^{\mu}-i \theta \Gamma^{\mu} \partial_{1} \theta$. The full set of constraints can be written in the form

$$
\begin{aligned}
& \left(p_{g}\right)_{a b}=0, \quad p_{\phi}=0, \quad p_{A_{0}}^{\mu}=0, \quad p_{\chi 0 \alpha}=0 ; \\
& \left(p^{\mu}{ }_{A_{1}}\right)^{2}=0, \quad \partial_{1} p^{\mu}{ }_{A_{1}}=0 ; \\
& p_{\Lambda}^{\mu}=0, \quad p^{\mu}{ }_{A_{1}}-\Lambda^{\mu}=0 ; \\
& H_{1} \equiv\left(\hat{p}^{\mu}+\Pi_{1}^{\mu}\right)^{2}-4 L_{\alpha} \partial_{1} \theta_{\alpha}=0, \quad H_{2} \equiv\left(\hat{p}^{\mu}-\Pi_{1}^{\mu}\right)^{2}=0 ; \\
& L_{\alpha} \equiv p_{\theta \alpha}-\partial_{1} p_{\chi 1 \alpha}-i\left(p^{\mu}+\Pi_{1}^{\mu}\right)\left(\theta \Gamma^{\mu}\right)_{\alpha}+i p_{A_{1} \mu}\left(\partial_{1} \theta \Gamma^{\mu}\right)_{\alpha}=0 ; \\
& p_{\chi_{1} \alpha}=0, \quad p_{A_{1} \mu}\left(\partial_{1} \theta-\chi_{1}\right)^{\beta} \Gamma^{\mu}{ }_{\beta \alpha}=0,
\end{aligned}
$$

where some of the initial constraints were exchanged on equivalent ones to simplify the Poisson brackets algebra. There are 1CC in Eqs. (28.a), (28.b), (28.d) and a trivial pair of $2 \mathrm{CC}$ in Eq. (28.c). Among 11 equations (28.b) only 10 are functionally independent, due to the identity $\partial_{1}\left[p_{A_{1}}^{2}\right]-2 p_{A_{1}}^{\mu}\left[\partial_{1} p_{A_{1} \mu}\right] \equiv 0$. Poisson brackets of the constraints (28.d), (28.e) are identical to those the GS superstring [1,5], in particular, $\left\{L_{\alpha}, L_{\beta}\right\}=2 i\left(\hat{p}^{\mu}+\right.$ $\left.\Pi_{1}^{\mu}\right) \Gamma^{\mu}{ }_{\alpha \beta} \delta\left(\sigma-\sigma^{\prime}\right)$. At last, the constraints (28.f) can be represented in equivalent form

$$
\begin{aligned}
& p_{A_{1} \mu} \tilde{\Gamma}^{\mu} p_{\chi 1}=0 ; \\
& \left(\hat{p}^{\mu}+\Pi_{1}^{\mu}\right) \tilde{\Gamma}^{\mu} p_{\chi 1}=0 ; \\
& p_{A_{1} \mu}\left(\partial_{1} \theta-\chi_{1}\right) \Gamma^{\mu}=0,
\end{aligned}
$$

with 8 independent 1CC among Eqs. (29) and 8+8 independent 2CC among Eqs. (30).

To investigate dynamics of the theory, we pass to light-cone coordinates $\left(x^{\mu} \rightarrow\right.$ $\left.\left(x^{+}, x^{-}, x^{i}\right), i=1, \ldots, 8, \theta^{\alpha} \rightarrow\left(\theta_{a}, \bar{\theta}_{\dot{a}}\right), a, \dot{a}=1, \ldots, 8\right)$, write out equations of motion for all variables with the help of Eq. (27), take into account the full constraints system (28), and impose gauge fixing conditions to 1CC. A self-consistent gauge choice is

$$
\begin{aligned}
& g^{\alpha \beta}=\eta^{\alpha \beta}, \quad \phi=1 / 2, \quad A_{0}^{\mu}=0, \quad \chi_{0}^{\alpha}=0, \\
& \theta_{a}=0, \quad \partial_{1} \bar{\theta}_{\dot{a}}-\bar{\chi}_{1 \dot{a}}=0, \\
& A_{1}^{-}=\tau, \quad A_{1}^{+}=A_{1}^{i}=0, \\
& x^{+}=-P^{+} \tau, \quad p^{+}=P^{+}=\mathrm{const} \neq 0 .
\end{aligned}
$$

After tedious calculations [18] one gets exactly the GS superstring dynamics for $x^{i}, p^{i}, \bar{\theta}_{\dot{a}}$ variables

$$
\partial_{0} x^{i}=-p^{i}, \quad \partial_{0} p^{i}=-\partial_{1} \partial_{1} x^{i}, \quad\left(\partial_{0}+\partial_{1}\right) \bar{\theta}_{\dot{a}}=0,
$$

while all other variables and Lagrange multipliers are expressed through them by means of algebraic equations. Note that in the gauge chosen, the relations $p_{A_{1}}^{-}=1, p_{A_{1}}{ }^{+}=p_{A_{1}}{ }^{i}=0$, $\hat{p}^{+}+\Pi_{1}^{+}=P^{+} \neq 0$, or, in covariant form

$$
p_{A_{1} \mu}\left(\hat{p}^{\mu}+\Pi_{1}^{\mu}\right) \neq 0
$$


hold. Return now to constraints (28.e), (29), (30), and rewrite them in equivalent form with the use of Eq. (33):

$$
\begin{aligned}
& \left(\hat{p}_{\mu}+\Pi_{1 \mu}\right) \tilde{\Gamma}^{\mu} L+p_{A_{1} \mu} \tilde{\Gamma}^{\mu} p_{\chi 1}=0 \\
& p_{A_{1} \mu} \tilde{\Gamma}^{\mu} L+\left(\hat{p}_{\mu}+\Pi_{1 \mu}\right) \tilde{\Gamma}^{\mu} p_{\chi 1}=0 \\
& p_{A_{1} \mu}\left(\partial_{1} \theta-\chi_{1}\right) \Gamma^{\mu}=0
\end{aligned}
$$

As a result, for the modified formulation of the GS superstring (23) fermionic constraints are splitted into first and second class (Eqs. (34) and (35), respectively) in a manifestly covariant way, so that the $1 \mathrm{CC}$ are irreducible.

An interesting peculiarity of the presented formulation is that it possesses $16+8$ fermionic reducible symmetries (25), (26), while first class constraints in the Hamiltonian formalism turn out to be irreducible. The reason is that except $16+8$ primary $1 \mathrm{CC}$ $p_{\chi_{0}}=0, \Lambda_{\mu} \tilde{\Gamma}^{\mu} p_{\chi_{1}}=0$, corresponding to the symmetries, there appear 8 secondary $1 \mathrm{CC}$ $\left(\hat{p}_{\mu}+\Pi_{1 \mu}\right) \tilde{\Gamma}^{\mu} L=0$. The two reducible sets are simply combined into irreducible one in the resultant system (34). This situation is opposite to the case of Siegel superparticle in the initial formulation $[12,13]$ for that symmetries are irreducible, while in the Hamiltonian formalism there arise reducible secondary first class constraints.

$D=10, N=1$ Casalbuoni-Brink-Schwarz superparticle. In this case we consider the following action:

$$
S=\int d \tau\left[-\frac{1}{2 e} \Pi^{\mu} \Pi_{\mu}-\omega-i \Lambda_{\mu} \chi_{1} \Gamma^{\mu}\left(\chi_{0}+\dot{\theta}\right)-\phi \Lambda^{2}\right]
$$

where $\Pi^{\mu} \equiv \dot{x}^{\mu}-i \theta \Gamma^{\mu} \dot{\theta}+\omega \Lambda^{\mu}$. The variables $\Lambda^{\mu}$ and $\chi_{1}{ }^{\alpha}$ turn out to be essential for realization of the supplementation scheme, while the variables $\omega, \phi \chi_{0}{ }^{\alpha}$ are in fact Lagrange multipliers which will supply appearance of the necessary constraints (3), (6). Similarly to previous case, there are reducible fermionic symmetries with $8+8+8$ intrinsic parameters

$$
\begin{aligned}
& \delta \theta^{\alpha}=\Pi_{\mu} \tilde{\Gamma}^{\mu \alpha \beta} \kappa_{\beta}, \quad \delta x^{\mu}=i \theta \Gamma^{\mu} \delta \theta, \\
& \delta e=-2 i e \dot{\theta} \kappa, \quad \delta \chi_{0}=-(\delta \theta)^{\prime} ; \\
& \delta \chi_{0}{ }^{\alpha}=\Lambda_{\mu} \tilde{\Gamma}^{\mu \alpha \beta} S_{0 \beta}, \quad \delta \phi=i \chi_{1}{ }^{\alpha} S_{0 \alpha} ; \\
& \delta \chi_{1}{ }^{\alpha}=\Lambda_{\mu} \tilde{\Gamma}^{\mu \alpha \beta} S_{1 \beta}, \quad \delta \phi=i S_{1 \alpha}\left(\chi_{0}+\dot{\theta}\right)^{\alpha} ;
\end{aligned}
$$

that exactly correspond to the independent primary first class constraints of the model (see below).

Remarkably, the action (36) leads only to the desired constraints (3), (6) for the variables $\Lambda^{\mu}$ and $\chi_{1}{ }^{\alpha}$ and does not imply any other constraints as it could be expected. We give here a detailed discussion of the Dirac-Bergmann algorithm for the model because some accuracy is necessary in treating its constraints system. The canonical Hamiltonian is

$$
H=-\frac{e}{2} p^{2}-\omega(p \Lambda-1)+i \Lambda_{\mu} \chi_{1} \Gamma^{\mu} \chi_{0}+\phi \Lambda^{2}+\xi_{0} p_{e}+\xi_{1} p_{\omega}+\xi_{2} p_{\phi}+
$$




$$
+\xi_{3 \mu} p_{\Lambda}{ }^{\mu}+p_{\chi 0} \sigma_{0}+p_{\chi 1} \sigma_{1}+L_{\alpha} \sigma_{3}{ }^{\alpha},
$$

where $\xi_{i}, \sigma_{i}$ are Lagrange multipliers corresponding to the primary constraints, and we denoted $L \equiv p_{\theta}-i p_{\mu} \theta \Gamma^{\mu}-i \Lambda_{\mu} \chi_{1} \Gamma^{\mu} \approx 0$. From requirement of preservation in time of the primary constraints, we get the secondary ones

$$
\begin{aligned}
& p^{2}=0, \quad \Lambda^{2}=0, \quad p \Lambda-1=0, \\
& \Lambda_{\mu}\left(\chi_{1} \Gamma^{\mu}\right)_{\alpha}=0 \text {, }
\end{aligned}
$$

and equations containing the Lagrange multipliers which can be represented in the form

$$
\begin{array}{ll}
\Lambda_{\mu} \Gamma^{\mu} \sigma_{1}=0, & \Lambda_{\mu} \Gamma^{\mu}\left(\chi_{0}-\sigma_{3}\right)=0, \\
p_{\mu} \Gamma^{\mu} \sigma_{3}=0, & -i \chi_{1} \Gamma^{\mu}\left(\chi_{0}-\sigma_{3}\right)+\omega p^{\mu}-2 \phi \Lambda^{\mu}=0 .
\end{array}
$$

(Multiplying the last equation by the $\Lambda_{\mu}$ we get as a consequence $\omega=0$ and the corresponding term may be omitted.) To analyze the system, note first that by virtue of Eq. (40), the following decompositions are possible:

$$
\chi_{0}=\chi+\tilde{\chi}, \quad \sigma_{3}=\sigma+\tilde{\sigma}
$$

where the corresponding components obey

$$
\Lambda_{\mu} \Gamma^{\mu} \chi=p_{\mu} \Gamma^{\mu} \tilde{\chi}=\Lambda_{\mu} \Gamma^{\mu} \sigma=p_{\mu} \Gamma^{\mu} \tilde{\sigma} .
$$

Then, one can verify that Eqs. (42) are equivalent to

$$
\begin{gathered}
\tilde{\sigma}=\tilde{\chi}, \quad \sigma=0, \quad \omega=0, \\
-i \chi_{1} \Gamma^{\mu} \chi-2 \phi \Lambda^{\mu}=0 .
\end{gathered}
$$

Moreover, passing to the light-cone coordinates and using $S O(8)$ notations for spinors it is easy to check that there is only one independent equation in Eqs. (46), if the conditions $\Lambda_{\mu} \chi_{1} \Gamma^{\mu}=0, \Lambda_{\mu} \Gamma^{\mu} \chi=0, \Lambda^{2}=0$ hold, namely: $-i \sqrt{2} \bar{\chi}_{1 \dot{a}} \bar{\chi}_{\dot{a}}-2 \phi \Lambda^{-}=0$. (Note that from the Eq. (40) it follows $\Lambda^{+} \neq 0$ or $\Lambda^{-} \neq 0$. For definiteness we choose the latter.)

The derived constraint forms a pair of second class constraints with $p_{\phi}=0$, and may be extracted from Eq. (42) in covariant way as follows: $-p_{\mu} \chi_{1} \Gamma^{\mu} \chi_{0}-2 \phi=0$. Finally, a full constraints system acquires the form

$$
\begin{aligned}
& p_{e}=p_{\omega}=\omega=0, \quad p_{\chi 0}=0 ; \\
& p^{2}=0, \quad p \Lambda-1=0, \quad \Lambda^{2}=0, \quad p_{\Lambda}{ }^{\mu}=0 ; \\
& L \equiv p_{\theta}-i p_{\mu} \theta \Gamma^{\mu}=0, \quad p_{\chi 1}=0, \quad \Lambda_{\mu} \chi_{1} \Gamma^{\mu}=0 ; \\
& p_{\phi}=0, \quad \Phi \equiv \phi+\frac{i}{2} p_{\mu} \chi_{1} \Gamma^{\mu} \chi_{0}=0 .
\end{aligned}
$$

The second constraint from Eq. (47.d) has nontrivial Poisson brackets with some of the constraints (47.a), (47.c). To avoid the obstacle, let us pass to the Dirac bracket associated with the pair (47.d)

$$
\{A, B\}_{D}=\{A, B\}-\left\{A, p_{\phi}\right\}\{\Phi, B\}+\{A, \Phi\}\left\{p_{\phi}, B\right\} .
$$


Then, it is admissible to consider the constraints as strong equalities and to resolve them. After that the variables $p_{\phi}, \phi$ can be dropped. As is seen from Eq. (48), the Dirac brackets for the remaining variables coincide with the Poisson ones.

As a result, for the model (36) we have got the desired constraints system (47.a-c). Actually, Eq. (47.c) can be rewritten in equivalent form

$$
\begin{aligned}
& p_{\mu} \tilde{\Gamma}^{\mu} L+\Lambda_{\mu} \tilde{\Gamma}^{\mu} p_{\chi 1}=0 \\
& \Lambda_{\mu} \tilde{\Gamma}^{\mu} L+p_{\mu} \tilde{\Gamma}^{\mu} p_{\chi 1}=0, \quad \Lambda_{\mu} \chi_{1} \Gamma^{\mu}=0
\end{aligned}
$$

with the $1 \mathrm{CC}$ (49.a) being irreducible.

Dynamics of the model may be analyzed along the same lines as has been done for the GS superstring above. Repeating all the needed steps, one can verify that physical sector and corresponding equations of motion of the modified formulation (36) are exactly the same as those of CBS superparticle.

Siegel superparticle. Within the framework of developed supplementation scheme, this is the most interesting example since a reducible $2 \mathrm{CC}$ are absent in the initial formulation $[12,13]$. As has been shown before, constraints system of the enlarged formulation for this case will include irreducible first and second class constraints only. The action, which reproduces the desired constraints system looks as follows:

$$
\begin{aligned}
S= & \int d \tau\left[-\frac{1}{2 e} \Pi^{\mu} \Pi_{\mu}+i \dot{\theta}_{R} \theta_{L}-i \Lambda_{\mu} \chi_{1} \Gamma^{\mu}\left(\chi_{0}+\dot{\theta}_{R}\right)-\right. \\
& \left.-\omega_{0}-\phi \Lambda^{2}-\omega_{2}\left(C^{2}-1\right)-\omega_{3}(C \Lambda)\right]
\end{aligned}
$$

where $\Pi^{\mu} \equiv \dot{x}^{\mu}-i \theta_{R} \Gamma^{\mu} \dot{\theta}_{R}+i \psi_{L} \tilde{\Gamma}^{\mu} \theta_{L}+\omega_{0} \Lambda^{\mu}+\omega_{1} C^{\mu}$, and $\theta_{R}^{\alpha}, \theta_{L \alpha}$ are Majorana-Weyl spinors of opposite chirality. The theory is invariant under the following generalization of Siegel $k$-symmetry:

$$
\begin{aligned}
& \delta \theta_{R}^{\alpha}=\frac{1}{e} \Pi_{\mu}\left(\tilde{\Gamma}^{\mu} \kappa_{L}\right)^{\alpha}, \quad \delta \theta_{L \alpha}=-\frac{2}{e^{2}} \kappa_{L \alpha} \Pi^{2}, \\
& \delta X^{\mu}=i \theta_{L} \tilde{\Gamma}^{\mu} \kappa_{L}+i \theta_{R} \Gamma^{\mu} \delta \theta_{R}, \\
& \delta e=-\frac{4 i}{e} \Pi_{\mu} \psi_{L} \tilde{\Gamma}^{\mu} \kappa_{L}, \quad \delta \psi_{L \alpha}=\dot{\kappa}_{L \alpha}, \quad \delta \chi_{0}{ }^{\alpha}=-\delta \theta_{R}{ }^{\alpha},
\end{aligned}
$$

that is irreducible as in the initial formulation $[12,13]$ as well as under a pair of reducible symmetries analogous to Eq. (38) of the previous case

$$
\begin{array}{ll}
\delta \chi_{0}{ }^{\alpha}=\Lambda_{\mu} \tilde{\Gamma}^{\mu \alpha \beta} S_{0 \beta}, \quad \delta \phi=i \chi_{1}{ }^{\alpha} S_{0 \alpha} \\
\delta \chi_{1}{ }^{\alpha}=\Lambda_{\mu} S_{1 \beta} \tilde{\Gamma}^{\mu \alpha \beta}, \quad \delta \phi=i S_{1 \alpha}\left(\chi_{1}+\dot{\theta}_{R}\right)^{\alpha}
\end{array}
$$

Canonical analysis for the model turns out to be very similar to that of the previous case. So, we present and discuss only the final answer for the full system of constraints

$$
\begin{aligned}
& p_{\omega i}=0, \quad \omega_{i}=0, \quad i=0,1,2,3 ; \\
& p_{\phi}=0, \quad \phi+\frac{i}{2} p_{\mu} \chi_{1} \Gamma^{\mu}\left(\chi_{0}-p_{\nu} \tilde{\Gamma}^{\nu} \psi_{L}\right)=0 ;
\end{aligned}
$$




$$
\begin{aligned}
& p_{\chi 0 \alpha}=0, \quad p_{\psi}{ }^{\alpha}=0, \quad \Lambda_{\mu} \Gamma^{\mu}\left(\chi_{0}-p_{\nu} \tilde{\Gamma}^{\nu} \psi_{L}\right)=0 ; \\
& p_{L}{ }^{\alpha}=0, p_{R \alpha}-i p_{\mu}\left(\theta_{R} \Gamma^{\mu}\right)_{\alpha}-i \theta_{L \alpha}=0 ; \\
& p_{e}=0, \quad p_{\Lambda}^{\mu}=0, \quad p C^{\mu}=0, \quad \Lambda^{2}=0, \\
& p \Lambda-1=0, \quad C^{2}-1=0, \quad p C=0, \quad C \Lambda=0 ; \\
& p_{\mu}\left(p_{R} \tilde{\Gamma}^{\mu}\right)^{\alpha}=0, \quad p_{\chi 1 \alpha}=0, \quad \Lambda_{\mu}\left(\chi_{1} \Gamma^{\mu}\right)_{\alpha}=0 .
\end{aligned}
$$

Here, we have a set of trivial 2CC (53.a); a pair of 2CC (53.d) which is the same as for the Siegel superparticle in the initial formulation [12, 13], and a pair of $2 \mathrm{CC}$ (53.b) that may be treated similarly to Eq. (47.d). Constraints system (53.c) consists of 24 independent $1 \mathrm{CC}$ and 16 independent 2CC which may be covariantly separated as follows:

$$
\begin{aligned}
& p_{\mu} \Gamma^{\mu} p_{\psi}=0, \quad \Lambda_{\mu} \tilde{\Gamma}^{\mu} p_{\chi 0}=0, \quad \Lambda_{\mu} \Gamma^{\mu}\left(p_{\nu} \tilde{\Gamma}^{\nu} p_{\chi 0}+p_{\psi}\right)=0 \\
& \Lambda_{\mu} \Gamma^{\mu}\left(p_{\nu} \tilde{\Gamma}^{\nu} p_{\chi 0}-p_{\psi}\right)=0, \quad \Lambda_{\mu} \Gamma^{\mu}\left(\chi 0-p_{\mu} \tilde{\Gamma}^{\mu} \psi_{L}\right)=0 .
\end{aligned}
$$

Choosing now gauge fixing conditions to $1 \mathrm{CC}$ (54.a) in the form

$$
\Lambda_{\mu} \tilde{\Gamma}^{\mu} \psi_{L}=0, \quad p_{\mu} \Gamma^{\mu} \chi_{0}=0, \quad \Lambda_{\mu} \Gamma^{\mu}\left(\chi_{0}+p_{\nu} \tilde{\Gamma}^{\nu} \psi_{L}\right)=0,
$$

one can check that the full system of equations (54.a), (54.b), and (55) is equivalent to $p_{\psi}=0, \psi_{L}=0, p_{\chi 0}=0, \chi_{0}=0$. Assuming the gauge has been imposed, let us pass to the Dirac bracket associated with the constraints $(53 . \mathrm{a}-\mathrm{c})$. Then the corresponding variables may be neglected, while the Dirac brackets for the remaining variables exactly coincide with the Poisson ones. The remaining constraints allow us to realize the supplementation scheme for reducible constraints $p_{\mu}\left(p_{R} \tilde{\Gamma}^{\mu}\right)=0$ which has been presented in the initial formulation. Actually, by virtue of Eqs. (53.e) one can combine fermionic constraints (53.f) into irreducible sets as follows:

$$
\begin{aligned}
& \Phi^{\alpha} \equiv p_{\mu}\left(p_{R} \tilde{\Gamma}^{\mu}\right)^{\alpha}+\Lambda_{\mu}\left(\tilde{\Gamma}^{\mu} p_{\chi 1}\right)^{\alpha}=0, \\
& G_{\alpha} \equiv \Lambda_{\mu}\left(\Gamma^{\mu} \chi_{1}\right)^{\alpha}+C_{\mu} p_{\nu}\left(\Gamma^{\mu} \tilde{\Gamma}^{\nu} p_{\chi 1}\right)_{\alpha}=0 .
\end{aligned}
$$

Here we have 16 irreducible $1 \mathrm{CC} \Phi^{\alpha}=0$ and 16 irreducible $2 \mathrm{CC} G_{\alpha}=0$ with the bracket $\left\{G_{\alpha}, G_{\beta}\right\}=-2 C_{\mu} \Gamma_{\alpha \beta}^{\mu}$.

Note in conclusion that for the case of the Lorentz group $S O(1,8)$ there exists only one inequivalent spinor representation of minimal dimension (the above mentioned matrix $z^{\alpha \beta}$ may be used for lowering and raising spinor indices). It means, in particular, that for $D=9$ Siegel superparticle it is not necessary to introduce variables $\Lambda^{\mu}, C^{\mu}$. This fact may extremely simplify a task of quantum realization of the scheme suggested.

\section{References}

[1] M.B. Green and J.H. Schwarz, Phys. Lett. B 136 (1984) 367.

[2] L. Brink and J.H. Schwarz, Phys. Lett. B 100 (1981) 310;

Casalbuoni R., Nuovo Cim. A 33, (1976) 389. 
[3] I.A. Batalin and I.V. Tyutin, Nucl. Phys. B 381 (1992) 619; Phys. Lett. B 317 (1993) 354; Mod. Phys. Lett. A 8 (1993) 3757.

[4] A.A. Deriglazov, A.V. Galajinsky, and S.L. Lyakhovich, hep-th/9512036, 21 p, subm. to Nucl. Phys. B.

[5] J.M. Evans, Phys. Lett. B 233 (1989) 307.

[6] J.M. Evans, Nucl. Phys. B 331 (1990) 711.

[7] L. Brink, M. Henneaux, and C. Teitelboim, Nucl. Phys. B 293 (1987) 505.

[8] F. Ebler, M. Hatsuda, E. Laenen, W. Siegel, J. Yamron, T. Kimura, and A. Micovic, Nucl. Phys. B 364 (1991) 67.

[9] M.B. Green and C.M. Hull, Nucl. Phys. B 344 (1990) 115; Mod. Phys. Lett. A 5 (1990) 1399.

[10] I.A. Batalin and E.S. Fradkin, Phys. Lett. B 122 (1983) 157; Lett. Nuovo Cim. 38 (1983) 393.

[11] W. Siegel, Nucl. Phys. B 263 (1985) 93.

[12] W. Siegel, Class. Quant. Grav., 2 (1985) 95.

[13] A.A. Deriglazov and A.V. Galajinsky, Mod. Phys. Lett. A 9 (1994) 3445.

[14] E. Nissimov, S. Pacheva, and S. Solomon, Nucl. Phys. B 296 (1988) 462; 297 (1988) 349; 299 (1988) 183.

[15] A.S. Galperin, P.S. Howe, and K.S. Stelle, Nucl. Phys. B 368 (1992) 248.

[16] A.S. Galperin and E. Sokachev, Phys. Rev. D 46 (1992) 714.

[17] Y. Eisenberg, Phys. Lett. B 276 (1992) 325.

[18] A.A. Deriglazov and A.V. Galajinsky, hep-th/9604006, 14 p.

[19] P.A.M. Dirac, Lectures on quantum mechanics. Yeshiva University (Academic Press, New York 1964).

[20] W. Siegel, Phys. Lett. B 128 (1983) 397. 